\title{
¿Qué es el populismo? Definición de los usuarios de Twitter durante la campaña electoral presidencial mexicana 2017- 2018
}

What is populism? Twitter users' definition during the Mexican presidential election campaign 2017-2018

\author{
Rubén Flores González \\ Universidad Veracruzana. Centro de Estudios de Opinión y Análisis \\ Orcid http://orcid.org/oooo-0002-2415-6742 \\ rubeflores@uv.mx
}

Resumen: Desde la academia se ha definido al populismo como ideología, estrategia, discurso, y encuadre de comunicación. Si bien se han alcanzado algunos consensos incluso entre estas diferentes formas de conceptualizar al término -la narrativa de confrontación entre el pueblo, y la élite, por ejemplo- para hacer estas definiciones los académicos se han basado en modelos de líderes o gobiernos que primero recibieron la denominación de populista de parte de la prensa o de la ciudadanía. Por lo tanto, se hace necesario reflexionar respecto a cómo se define al populismo desde el debate político cotidiano. Con este propósito se realizó un análisis de contenido cualitativo a una muestra de 4,339 tuits con menciones sobre el populismo, publicados en el contexto de las elecciones presidenciales mexicanas. Se observa que los usuarios de Twitter describieron al populismo como ideología y como estrategia política. Ambas definiciones se hicieron desde la oposición afectiva al populismo, y se utilizaron para descalificar a algunos de los candidatos en la contienda.

Palabras clave: Populismo, Twitter, ideología, elecciones, comunicación política

\begin{abstract}
Scholars have defined populism as an ideology, a strategy, a discourse, and a communication frame. Some agreements have been reached among these various conceptualizations. However, scholars seem to have defined populism through the observation of leaders and governments that already were called populist by the press or some citizens. Thus, a reflection is needed regarding the construction of the populist-concept in everyday political debates. With this purpose, a qualitative content analysis was performed in a sample of 4,339 tweets mentioning populism, in the context of the Mexican presidential elections. It was observed that Twitter users described populism as an ideology and as a political strategy. Both definitions were made from an affective opposition standpoint, and so were used to discredit some of the presidential candidates.
\end{abstract}

Keywords: Populism, Twitter, ideology, elections, political communication

Fecha de recepción: 04/06/2019

Fecha de aprobación: 13/09/2019

Cómo citar este artículo / How to cite this paper: Flores González, R. (2019). ¿Qué es el populismo? Definición de los usuarios de Twitter durante la campaña electoral presidencial mexicana 2017-2018. Revista de Comunicación Política, 1, 11-29. doi:10.29105/rcp1-1 


\section{Introducción}

El populismo es un fenómeno que ha modificado de forma contundente el panorama político de occidente, hasta hace poco dominado por las democracias neoliberales. Mencionado por muchos, su observación es compleja debido a la diversidad de actores; líderes, partidos, gobiernos, que en un momento u otro han sido calificados como populistas.

Poco a poco en el campo académico se han alcanzado ciertos consensos: el discurso populista divide a la sociedad en dos bandos. Un bando que es el pueblo y otro bando que es la élite. El pueblo es oprimido por la élite pese a que de éste emana el poder legítimo (Canovan, 2004; Laclau, 2004; Marchlewska, Cichocka, Panayiotou, Castellanos, \& Batayneh, 2018). En esta narrativa de confrontación, las instituciones tradicionales de la democracia no pueden representar al pueblo -porque están al servicio de la élite- y ese lugar lo asume un líder o lideresa carismático. Este líder o lideresa es depositario del capital político que emana de una gran cantidad de seguidores que están poco organizados entre sí. Debido a que el poder del líder surge de sus seguidores, éste busca por todos los medios estar en contacto con ellos a través de la organización de mítines, plebiscitos, elecciones, o referéndums (Mazzoleni, 2008; Urbinati, 2019; Weyland, 2001). En el plano económico, los gobiernos populistas se han caracterizado por debilitar a los órganos autónomos que en las democracias liberales determinan las políticas económicas de largo plazo, tales como lo bancos centrales. Por otra parte, también han regulado la inversión privada de manera caprichosa, ahuyentándola eventualmente (Rodrik, 2018).

Siendo un término que ha sido hasta cierto punto sobreexplotado en la política y en diversos medios de comunicación; el populismo se ha utilizado para designar alternativamente, una ideología, una lógica política, un discurso y una estrategia (Moffit \& Tormey, 2014). Weyland (2001) y Barr (2018) mencionan que el populismo es una estrategia para obtener y mantener el poder político, caracterizada porque la capacidad de poder esgrimida por un líder procede del apoyo que recibe de un gran número de personas, con quienes mantiene un contacto fluido y poco organizado. Como ideología, el populismo promueve de forma superficial la reivindicación de un conjunto indeterminado llamado pueblo, de otro conjunto indeterminado denominado élite. Debido a esta vaguedad, el populismo se vincula a otras ideologías para complementar su propuesta política (Freeden, 2017) y en congruencia con esto, el radicalismo ideológico es una característica de las plataformas políticas señaladas como populistas (Jacobs \& Spierings, 2018; March \& Mudde, 2005; Mudde, 2010; Ramiro \& Gómez, 2017). Si es de izquierda, el enemigo del pueblo es una élite económica; si es de derecha, dentro de los enemigos también se cuenta a las minorías y a los extranjeros, y se exacerba el nacionalismo (Lacatus, 2019; Katsambekis \& Stavrakakis, 2017). Esto, si bien va en detrimento de un sistema democrático constitucional y representativo, no es contrario a los fundamentos esenciales de la democracia, puesto que el poder que el líder populista concentra proviene del apoyo de una gran masa de seguidores (Urbinati, 2019). Para hacer más efectiva esta concentración de poder, se debilita a las 
instituciones autónomas que son intermediarias entre el estado y el "pueblo" tales como los partidos políticos, o que impiden al líder hacer efectivos sus beneficios directos al pueblo. Esta misma lógica, de eliminación de intermediarios se sigue en el modelo de comunicación de líderes y gobiernos populistas.

\section{El populismo en la comunicación}

En contraposición a la definición del populismo como ideología, se ha propuesto que el populismo es un discurso identificable a partir de su encuadre comunicativo. Esto es, tanto el pueblo como la élite no señalan por sí a un colectivo en particular a partir de características precisas, sino que son encuadres vacíos de contenido que pueden aplicarse a diferentes conjuntos según se presenten las necesidades políticas. Un sentido de urgencia respecto a un problema, el estilo confrontativo y el uso del miedo completarían ocasionalmente las características de este encuadre (Aslanidis, 2015; Casero-Ripollés, Sinte-Olivella, \& Franch, 2017; Reinemann, Aalberg, Esser, Strömback, \& de Vresse, 2016).

Visualizar el populismo como un encuadre nos ayudaría a entender por qué existen populismos de izquierda y de derecha. Este encuadre, como se puede observar, resalta por su simplismo, lo que impone cierta lógica en cuanto a su producción y posibilidades de divulgación. Facilita, por ejemplo, la eliminación de intermediarios en la relación entre el líder populista y la masa. En los inicios del populismo, cuando los medios tradicionales seguían siendo la única vía para difundir un mensaje masivamente, los líderes utilizaron instrumentalmente a los medios para que se hablara de ellos y sus propuestas sin necesidad de pagar por anuncios publicitarios. Consiguieron lo anterior mediante discursos provocadores que inevitablemente se convertían en noticia. Satisfacían la necesidad de los medios de ofrecer a las audiencias contenidos espectaculares, especialmente relacionados con la política (Mazzoleni, 2008); otra de sus estrategias consistió en atacar a los medios informativos tradicionales como representantes de los intereses de la élite y no de los del pueblo. La confrontación con los medios rindió frutos en cuanto a la cobertura de los líderes que se destacaron a inicios del siglo XXI (Stewart, Mazzoleni, \& Horsfield, 2003).

La llegada de las redes sociales abrió un panorama prometedor para los líderes populistas, que vieron en esta plataforma una oportunidad más para cimentar el contacto directo con sus seguidores. A esto ayudaron dos situaciones: una de ellas fue que las redes sociales se presentaron como un espacio propicio para la comunicación horizontal, no mediada por las élites, y que por lo mismo transpiraba cierto espíritu de rebeldía. Las redes sociales fueron fundamentales en la organización de algunos movimientos civiles con cierta relevancia como la Primavera Árabe, el Occupy Wall Street, la campaña de Barack Obama, o el movimiento estudiantil \#YoSoy132. Por otra parte, la emergencia de las redes sociales coincidió con una crisis del sistema neoliberal y de las democracias a nivel global. 
La incertidumbre económica y el desencanto con la política dieron sustento a la narrativa de un pueblo agraviado por una élite (Gerbaudo, 2018).

\section{Populismo en redes sociales}

Parece indiscutible que las redes sociales han sido un vehículo propicio para la diseminación de los mensajes populistas y el posicionamiento de sus líderes a nivel mundial (e.g. Lacatus, 2019). Sin embargo, existe una discusión respecto a los métodos y los efectos del uso de estas plataformas. Contrario a la postura de que las redes sociales son el medio favorito para la diseminación de discursos populistas (Gerbaudo, 2018), también se ha observado un uso complementario redes-medios masivos en campañas electorales modernas por parte de candidatos con esa denominación (Groshek \& Koc-Michalska 2017). Para explicar lo anterior, basta recordar que en la actualidad varios medios masivos son asimismo usuarios de redes sociales y que, de igual forma, no todos los medios tradicionales son exigentes en cuanto a la selección y presentación de información objetiva o equilibrada.

No se ha observado de forma consistente el que líderes populistas hagan un mayor número de publicaciones en redes sociales que otro tipo de líderes (e.g. Hernández, 2019). No obstante, se ha reportado que estos líderes utilizan las redes sociales con un menor grado de compromiso político, y con mayor selectividad en cuanto a los integrantes de su red de contactos, contribuyendo así a la generación de cámaras de eco (Jacobs \& Spierings, 2018). En cierta contraposición con lo anterior, también se ha observado que, durante una campaña electoral, la presencia de discursos populistas en las redes aumenta la posibilidad de que existan discusiones entre personas con diversos posicionamientos ideológicos (Groshek \& Koc-Michalska 2017).

Los contenidos populistas en redes sociales son consistentes con la definición del populismo como discurso. Son de carácter confrontativo, e integran los elementos pueblo, élite, rechazo a los extraños -en caso de populismos de derecha-, y la narrativa de que existe una crisis (Casero-Ripollés, et al., 2017; Ernst, Engesser, Büchel, Blassnig, \& Esser, 2017). Haciendo énfasis en su carácter confrontativo, las publicaciones populistas en los medios sociales suelen atacar muy puntualmente a actores o personajes que son representativos de un posicionamiento ideológico opuesto (Van Kessel \& Castelein, 2016). En gobiernos populistas, estos ataques se han observado a partir de la acción coordinada de cuentas automatizadas y el uso de trolls (Bulut \& Yörük, 2017).

Finalmente, se ha señalado que las restricciones de contenido inherentes a la producción y consumo de las redes sociales son congruentes con la naturaleza fragmentaria del discurso populista (Engesser, Ernst, Esser, \& Büchel, 2017). Esto es, los mensajes populistas resaltan a partir de dos de sus características: ambigüedad y sencillez. La ambigüedad de la apelación al pueblo permite la inclusión en esta categoría de un amplio número de seguidores potenciales. La sencillez se refiere a que, si bien el populismo siempre se acompaña de un posicionamiento ideológico radical, el contenido 
ideológico del mensaje suele ser sobre-simplificado para que pueda ser comprendido por la mayor cantidad de personas posible. Las redes sociales más populares, como Facebook y Twitter, imponen un contenido de caracteres en la producción de los mensajes, a la vez que facilitan el consumo de contenidos concisos. En ese tenor, los mensajes populistas -ambiguos, sencillos y sensacionalistasson los más aptos para ser consumidos y reproducidos a través de estas plataformas.

\section{Populismo en el proceso electoral federal mexicano 2017- 2018}

En la elección participaron cuatro candidatos y una candidata. De estas cinco opciones, el candidato Andrés Manuel López Obrador (coalición MORENA-PT-PES) aspiraba a la presidencia por tercera vez. En algunas notas de medios nacionales, así como en algunas observaciones académicas, se resaltó que este personaje y su plataforma electoral eran "populistas” (Bedoya \& Colín, 2015; Bolívar, 2017; Gutiérrez, 2007; Marini, 2018). Sin embargo, dentro de la academia latinoamericana que ha abordado el tema de las elecciones presidenciales mexicanas, se habla del populismo a menudo relacionado con una ideología, específicamente como ideología de izquierda, antes que con un estilo de comunicación (Balderas \& Tapia, 2019; Bolívar, 2017; Loaeza, 2007). Probablemente esto se deba a la referencia que se tiene en Latinoamérica de un periodo en que hubo un auge de gobiernos de izquierda, de los cuáles varios fueron calificados como populistas (Castañeda, 2006; Freidenberg, 2007; Loaeza, 2007).

Resulta interesante explorar esta disyuntiva conceptual entre la academia latinoamericana, habituada al populismo de izquierda, con las academias norteamericanas y europeas, que han realizado observaciones sobre líderes y gobiernos calificados como populistas de derecha. También es curioso que la designación populista respecto a un personaje, plataforma o partido suela venir a menudo de una fuente externa. En el argot cotidiano, populismo es casi una mala palabra con la que se estigmatiza a un adversario político para denostarlo (e.g. Gutiérrez, 2007). Y si bien se han hecho observaciones respecto a los encuadres del populismo desde la academia y desde los medios de comunicación, la discusión política alrededor de este término nos brinda la oportunidad de determinar cuáles son las características que destacan los usuarios de las redes sociales para definir el populismo. En atención a esto, el presente estudio tiene como objetivo conocer cómo definieron al populismo algunos usuarios de Twitter en el contexto del proceso electoral federal mexicano 2017-2018. 


\section{Método}

Se realizó un análisis de contenido cualitativo de tuits publicados entre el 16 de octubre de 2017 y el 2 de julio de 2018, que mencionaran la palabra "populismo" y que mencionaran a alguno de los candidatos contendientes en la elección presidencial: Andrés Manuel López Obrador, Ricardo Anaya Cortés, José Antonio Meade Kuribeña, Jaime Rodríguez Calderón, y Margarita Zavala Gómez del Campo. Los siguientes pasos se tomaron para seleccionar el material de trabajo.

Se realizó una minería de tuits de lunes a viernes durante el periodo mencionado, utilizando el paquete rtweet (Kearney, 2016) en el ambiente de programación R. Se solicitaron 10,000 tuits diarios mencionando a cada candidato utilizando sus nombres de usuario oficiales en la red social como términos de búsqueda: @lopezobrador_, @JoseAMeadeK, @RicardoAnayaC, @JaimeRdzNL, y @Mzavalagc. No siempre fue posible, por fallos humanos o interrupciones en la conexión, recolectar 50,00o tuits diarios. Cabe recordar que la candidata Margarita Zavala renunció a su candidatura el 16 de mayo, por lo que a partir de esa fecha cesó la recolección de tuits mencionándola. Se recolectaron 1,957,760 tuits en total distribuidos como se indica en la Tabla 1.

Tabla 1. Distribución de tuits recolectados por candidatura

\begin{tabular}{|c|c|c|}
\hline Candidato & Tuits recolectados & $\%$ \\
\hline Andrés Manuel López Obrador & 400,146 & 20 \\
\hline José Antonio Meade Kuribeña & 473,732 & 24 \\
\hline Ricardo Anaya Cortés & 433,001 & 22 \\
\hline Jaime Rodríguez Calderón & 401,984 & 21 \\
\hline Margarita Zavala Gómez del Campo & 248,897 & 13 \\
\hline
\end{tabular}

Cabe señalar, que el presente estudio forma parte de un proyecto más amplio relacionado con la ideología, por lo que en un primer filtro se utilizaron los siguientes términos de búsqueda para detectar tuits con evidente contenido ideológico: ideología, izquierda, derecha, capitalista, capitalismo, socialista, socialismo, comunista, comunismo, neoliberal, populista, populismo, fascismo, fascista. Tras eliminar los tuits repetidos en esta segunda base de datos, los casos se redujeron a 9,707 publicaciones. De inmediato resaltó que, en estos resultados, las referencias al populismo -populismo, populista, populistas- destacaron por encima de los demás términos de búsqueda con 4,339 menciones.

Este resultado como antecedente fue una de las motivaciones para explorar los significados que los usuarios dieron al populismo. Para ello, se realizó un análisis de contenido, clasificando el 
texto de las publicaciones de acuerdo con categorías que se definieron a priori tomando en cuenta la conceptualización del populismo como una ideología. Las conceptualizaciones del populismo como comunicación y como estrategia no fueron tomadas en cuenta puesto que las unidades de análisis no eran fragmentos del discurso populista, o productos en sí de la actividad de algún líder populista, sino expresiones de los usuarios de la red social. Pese a lo anterior, se encontró en el análisis final que algunos de los tuits mencionaban al populismo como una estrategia y no como una ideología. Esta diferencia es explicada en la discusión. Asimismo, desde el punto de vista de los usuarios se encontraron características en el discurso de los tuits en las que se observan ciertas particularidades en la direccionalidad y el tono afectivo con el que se habla del populismo en política.

Por lo anterior, en la estructura de categorías que se menciona a continuación, se recolectó el posicionamiento sobre diferentes actores respecto al populismo esperando ver reflejado en estos posicionamientos una dicotomía entre el pueblo y las élites (Canovan, 2004; Laclau, 2004). De la misma forma, las categorías iniciales de equidad socioeconómica y de iniciativa privada y macroeconomía se propusieron anticipando la prevalencia del populismo de izquierda en la elección, el tipo de populismo más observado en América Latina (Castañeda, 2006; Freidenberg, 2007), en el que los temas económicos y de desigualdad social son más explotados (Lacatus, 2019); y puesto que uno de los candidatos tenía antecedentes de ser señalado como un político populista de izquierda (Bolívar, 2017). Inicialmente, las categorías propuestas para el análisis de los tuits se definieron como sigue:

Posicionamiento de candidatos. En esta categoría se incluyen textos en los que se señala explícitamente la posición política de cuando menos un candidato o candidata en relación con el populismo.

Posicionamiento de personajes u organizaciones. El discurso posiciona a personas u organizaciones que no son candidatos como representantes u opositores del populismo.

Posicionamiento internacional de estados o gobiernos. Se relaciona a un estado extranjero o a un gobierno extranjero como representante u opositor del populismo.

Equidad socioeconómica. Se menciona en el discurso una posición respecto a la asistencia social. Al apoyo a grupos más pobres, vulnerables o necesitados. Se hacen pronunciamientos respecto a la discriminación, el clasismo o el racismo. Se habla de acciones para reducir la brecha entre ricos y pobres, o para aumentarla.

Descripción ideológica. Se hace una descripción del populismo como ideología. Se menciona que los populistas son de una manera; que el populismo es tal cosa, que tiene ciertas consecuencias, o defiende ciertos valores. Se habla de las consecuencias del populismo.

Iniciativa privada y macroeconomía. Se habla específicamente sobre política económica, empresarios, empresas, facilidades para la iniciativa privada, obstáculos a empresas o iniciativa privada. Privatizaciones, o expropiaciones. 
Posterior a una primera prueba de clasificación del contenido, se agregó también la categoría de libertad de expresión, definida como sigue:

Libertad de expresión. Se habla de la suspensión, o violación del derecho a la libre expresión a partir de actos de autoridad. Se habla de represión o de censura. Se habla de la libertad de expresión en general, o de la promoción de la libertad de expresión.

A partir de una segunda exploración en la clasificación de los tuits, se decidió subordinar las categorías de iniciativa privada y macroeconomía, equidad socioeconómica, y libertad de expresión a la categoría descripción ideológica. Todas estas categorías se consideró que eran las que mejor contribuían a una definición conceptual del populismo, de acuerdo con los tuits observados. A éstas se agregó la categoría general, con el propósito de recolectar fragmentos de expresiones ideológicas que no tuvieran que ver con equidad o macroeconomía. En la práctica, esta subcategoría recopiló solamente el vínculo del populismo con términos ideológicos tradicionales como: derecha, izquierda, socialismo o comunismo. Por otra parte, se concibió otra categoría para subordinar a aquellas relacionadas con los posicionamientos en torno al populismo de los diferentes actores mencionados. En la Tabla 2 se presenta el desglose final de las categorías y subcategorías utilizadas, junto con el número de fragmentos de tuits que compusieron su corpus final.

Tabla 2. Categorías y subcategorías de análisis

\begin{tabular}{|c|c|c|}
\hline Categoría & Subcategoría & Fragmentos de tuits \\
\hline \multirow{4}{*}{ 1. Posicionamientos } & 1.1 Candidatos & 3,240 \\
\hline & 1.2 Personas & 192 \\
\hline & 1.3 Organizaciones & 312 \\
\hline & 1.4 Estados & 273 \\
\hline \multirow[t]{4}{*}{ 2. Descripción ideológica } & 2.1 Equidad socioeconómica & 519 \\
\hline & 2.2 IP y macroeconomía & 265 \\
\hline & 2.3 Libertad de expresión & 179 \\
\hline & 2.4 General & 231 \\
\hline
\end{tabular}

Con esta estructura, los fragmentos del texto de los tuits fueron clasificados en alguna de las subcategorías por dos personas capacitadas en el uso de las definiciones propuestas, formando un corpus para cada una de ellas. Estos corpus fueron leídos y se realizaron reflexiones finales de cada uno. Se consideraron las reflexiones finales de cada corpus y las relaciones entre ellos para proponer un modelo que describa cómo definieron al populismo los usuarios de Twitter. 


\section{Resultados}

\section{Posicionamiento de los candidatos}

El candidato que fue caracterizado mayormente como populista fue Andrés Manuel López Obrador. Sin embargo, no fue el único definido de esta manera. Esto puede verse en el número de tuits en que se hace referencia al populismo y que además mencionan a alguno de los candidatos (Figura 1).

Figura 1. Número de tuits sobre populismo en los que se mencionó a alguno de los candidatos

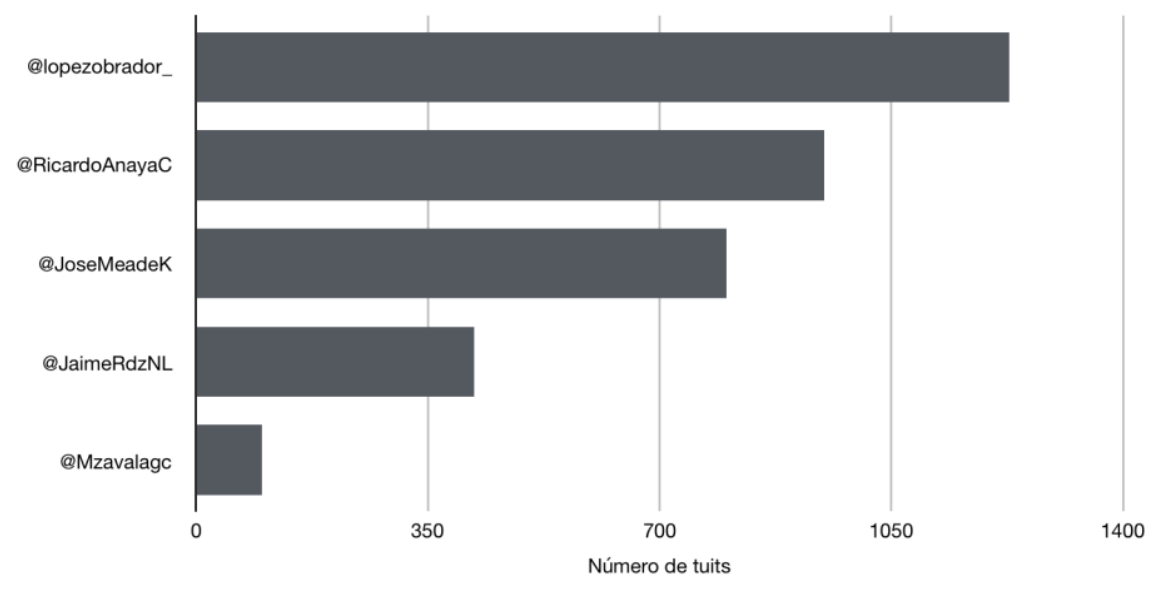

En ese sentido, hay que decir que los usuarios de Twitter encuadraron la elección ideológicamente como una lucha entre dos bandos: por una parte, el bando populista, mejor representado por Andrés Manuel López Obrador, y por otra parte el bando neoliberal, representado por el resto de los candidatos. Sin embargo, este hallazgo es de un análisis que no corresponde a los objetivos de esta publicación. Es necesario aclararlo porque, si bien Andrés Manuel López Obrador fue identificado como el representante del populismo, el resto de los candidatos también fueron etiquetados en algunas instancias de esta manera, sin que esa haya sido su característica principal según se observó en el estudio más amplio. Algunos ejemplos de esta identificación populista son los siguientes:

A6. iLos que no queremos a \#AMLO es porque no queremos retroceder...queremos que lo que ya se logró no lo destruya un mesías populista! La única opción el \#Votoutil \#VotoRazonado 
J114. @beltrandelrio POPULISMO, es ese q practican todos los candidatos, Anaya fingiendo dormir en el piso con su cobija d tianguis, Meade en una comunidad pobre del Edo de Mex, Obrador vestido de Chamula en mitin de Chiapas, el Bronco boleando zapatos...

Pese a esto, existen algunas diferencias fundamentales en cómo fue caracterizado el populismo de unos y de otros. Mientras que a Andrés Manuel López Obrador se le menciona como populista con cierta coherencia ideológica, el resto de los candidatos son descritos como populistas sólo de forma instrumental o estratégica. Esto es, se supone que el populismo de Andrés Manuel no es nuevo y que tendrá consecuencias en las políticas públicas de la nación de ganar la presidencia. El populismo del resto de los candidatos se focaliza sólo en las propuestas que realizan con el objetivo inmediato de ganar la elección, y por lo mismo se describe como una contradicción a su verdadera naturaleza ideológica. Esta caracterización de los candidatos como contradictorios en torno a su posición frente al populismo fue más frecuente respecto a Ricardo Anaya y José Meade.

J9. ¿Porque le teme Anaya, Meade y Zavala al populismo de AMLO? Creo que es muy fácil de entender, Obrador quiere invertir miles de millones en programas sociales, dinero que estas [emoticones de ratas] ya no se podrán robar.

J6o. @AngyieT @EnriqueOchoaR @JoseAMeadeKLLa \#LeyMeade o sea la \#LeyAtole no es otra cosa que una propuesta de hace mucho tiempo de \#YaSabenQuien. Que en \#AMLO la veían malísima y populista, pero en las ratas derechosas del \#PRI y del \#PAN son buenísimas y novedosas, según ellos. Pero qué creen? MEXICO YA NO LES CREE MÁS

Una muestra de que los usuarios de Twitter identificaron a los candidatos Jose Antonio Meade, Ricardo Anaya, Margarita Zavala, y Jaime Rodríguez como parte de un bloque opositor al populismo se observa en los múltiples llamados que les hicieron para formar una alianza electoral.

R601@RicardoAnayaC @JoseAMeadeK @Mzavalagc deberían pensar en una alianza para salvar al país del populismo que tanto daño ha hecho en América Latina. Hoy lloran Nicaragua y Venezuela.

B702. Para como veo las cosas; @JoseAMeadeK @RicardoAnayaC@Mzavalagcy @JaimeRdzNL es imperativo que los cuatro soliciten al INE una $2 a$ Vuelta Sólo así podremos asegurar que AMLO no gane. México está dividido Uds. deben unirnos contra el populismo. Sea quien sea el 20 lugar. Por favor 


\section{Definición de populismo}

En principio, el populismo fue definido como la acción del estado de regalar dinero, bienes o servicios, so pretexto de buscar la equidad socioeconómica, a un conjunto de personas que no merece dichos beneficios. Esto resulta, a decir de los usuarios, congruente con un posicionamiento ideológico de izquierda, socialista, o comunista.

A110. @AlmaPagana@MariRojas65 @lopezobrador_Eso no importa,pues el Peje va a mantener a los NINIS y los va a meter a la UNAM, a pesar de que no sepan escribir.

A183. @belindapop Cuidado, AMLO y equipo tienen ideas comunistas (Expropiacines), te van a quitar tus bienes que con esfuerzo conseguiste para darselos a gente que no tiene por floja, aun estamos a tiempo de parar a este loco, soy un simple ciudadano preocupado, no soy de la mafia del poder.

Esta acción afecta a empresarios y personas productivas, y se presume que el gasto social realizado de esta forma ocasionaría una catástrofe económica.

A802. El POPULISMO socialista está CONDENADO al fracaso, es comida para hoy y hambre y miseria para mañana. JAMÁS votaremos por AMLO y su Morena.

Por último, fue reiterativa la descripción del populismo como un sistema opuesto a la libertad de expresión a partir de una situación muy específica que se presentó durante la campaña: la supuesta producción de un documental en contra del populismo en América Latina, y las acciones que el candidato Andrés Manuel López Obrador habría tomado para evitar su presunta difusión.

A903. @lopezobrador_AMLO, me preocupa tu antidemocracia y autoritarismo. Por qué quieres censurar el documental de @NatGeo_la? \#amloNo \#amlopopulista

Posicionamiento de estados, personajes y organizaciones en torno al populismo

Se definió al populismo también a partir de los estados que presuntamente lo han experimentado, así como a una serie de personajes que lo respaldan o lo confrontan. La mayoría de las menciones se hicieron en torno a líderes y países que protagonizaron el giro a la izquierda en América Latina: 
A567. No cometamos el mismo error de Argentina, Venezuela o Brasil, eligieron gobiernos populistas y vean cómo les fue. Algo peor que la corrupción es el Populismo, lleva a la quiebra a un país. Pensemos seamos sensatos, reflexionemos. \#AMLO no es la solución.

B154. Goldenofman @Apuntesmios @mandarina4x4 Sí votas por un basura comunista si, estás pendejo, es preferible votar por el bronco o por nueva alianza. Ya estamos grandecitos pa estar creyendo en populistas del "pueblo", los latinoamericanos no aprenden, Castro en Cuba, Ortega en Nicaragua, Chávez en Venezuela, Evo en Bolivia...

Se resalta sobre todo la posición de algunos intelectuales y líderes de opinión en contra del populismo como Gloria Álvarez, Vargas Llosa y Enrique Krauze. Otro grupo de intelectuales y líderes de opinión son caracterizados como simpatizantes del populismo, pero no hay muchas referencias de esto último.

A178.@annhelasan @marinaocejo @AristeguiOnline @lopezobrador_Ya que hablas de libros lee El Engaño Populista de Axel Káiser y Gloria Álvarez, también puedes ver videos publicados por estos dos en YouTube donde explican que es ser populista y porque AMLO se caracteriza por serlo.

J124. @tatclouthier @CiroGomezL // Que oportuna y certeramente está hablando @EnriqueKrauze sobre la preocupante realidad de la mesiánica y populista personalidad de @lopezobrador_...Mis respetos para Krauze...

A781. Es tanta la obsesión por el poder y el odio de los "populistas de huarache" mexicanos, que Paco Ignacio Taibo II -gurú de @lopezobrador_ - adelantó los tiempos con sus revelaciones...@m_ebrard salió a callarlo, consciente de la estupidez.

En cuanto a las organizaciones, se destacó el rechazo de organismos empresariales a la posible implementación de políticas populistas.

A69o. \#Elecciones2018 @Coparmex respalda a Grupo México para "no votar por un gobierno populista"; @lopezobrador_se defiende y los llama traficantes de influencias https://t.co/WaUKoDjFab https://t.co/58VDLdmgNw

J707. @JoseAMeadeK Los populista Socialistas no ven el desarrollo del país, ni aprecian s los empresarios que generan empleos, ellos quieren todo gratis, con @JoseAMeadeK hay futuro, personas no partidos! 
Y en esta misma categoría, se relacionó a los principales partidos políticos -PRI, PAN y MORENA - con el populismo, tanto a partir de las propuestas de sus candidatos, como se mencionó, como por la operación de programas sociales con fines clientelares en las ocasiones en que algunos de sus militantes han formado parte del gobierno.

R923. @yors3247 @damianzepeda @ReneJuarez Aja! Y el Pri no regala despensas, tinacos y porqueria y media, y Anaya no esta dando tarjetas sin fondos? No quiere dar las tablets como Maduro? Eso también no es ser populista? Solo ven lo que Amlo hace pero el Pri y el Pan están del nabo por eso Nadie los quiere ya!

B340. "Ampliar programa Prospera (2 Millones de personas mas al programa)" "Becas 10o\% de Universidad para los que terminaron la Preparatoria" "Reducir la diferencia entre pobres y ricos" (Programas asistencialistas). Ah, pero el Populista es AMLO. Yo con Bronco.

B382.Esto será \#Populismo ? @VicenteFoxQue @FelipeCalderon @EPN @RicardoAnayaC@JoseAMeadeK Me acaban de robar mi lona de MORENA, con la que tenía tapado el cemento que me dió el PAN, para hacerle su base al tinaco que me dió el PRI. Avísenle al Bronco para que les moche la mano!!!

\section{Propuesta de modelo}

Se observa que, en el marco de la elección presidencial de 2018, el populismo en Twitter se definió a partir de las publicaciones de usuarios que manifestaron su oposición hacia éste. En congruencia con lo anterior, el término se utilizó para descalificar a alguno de los candidatos. Se habló del populismo como una ideología de izquierda y como una estrategia. Como ideología, se mencionó que el populismo busca convertir en política pública el otorgar gratuitamente bienes y servicios a personas que no los merecen con el fin de reducir la desigualdad socioeconómica. Sin embargo, según el ejemplo de los países del giro a la izquierda latinoamericano, esta política estaría destinada a llevar al estado a un colapso económico. Como estrategia, el populismo tiene una lógica instrumental, pues se refiere tanto a las propuestas como a las dádivas de campaña, que se otorgaron con el propósito -perverso y engañoso- de ganar la elección. Es necesario resaltar que tanto la definición ideológica como la estratégica se fundamentan en el concepto de regalar algo a alguien que no lo merece con un resultado socialmente negativo. 
Figura 2. Propuesta de modelo descriptivo de populismo de acuerdo con las menciones del término proporcionadas por usuarios de Twitter

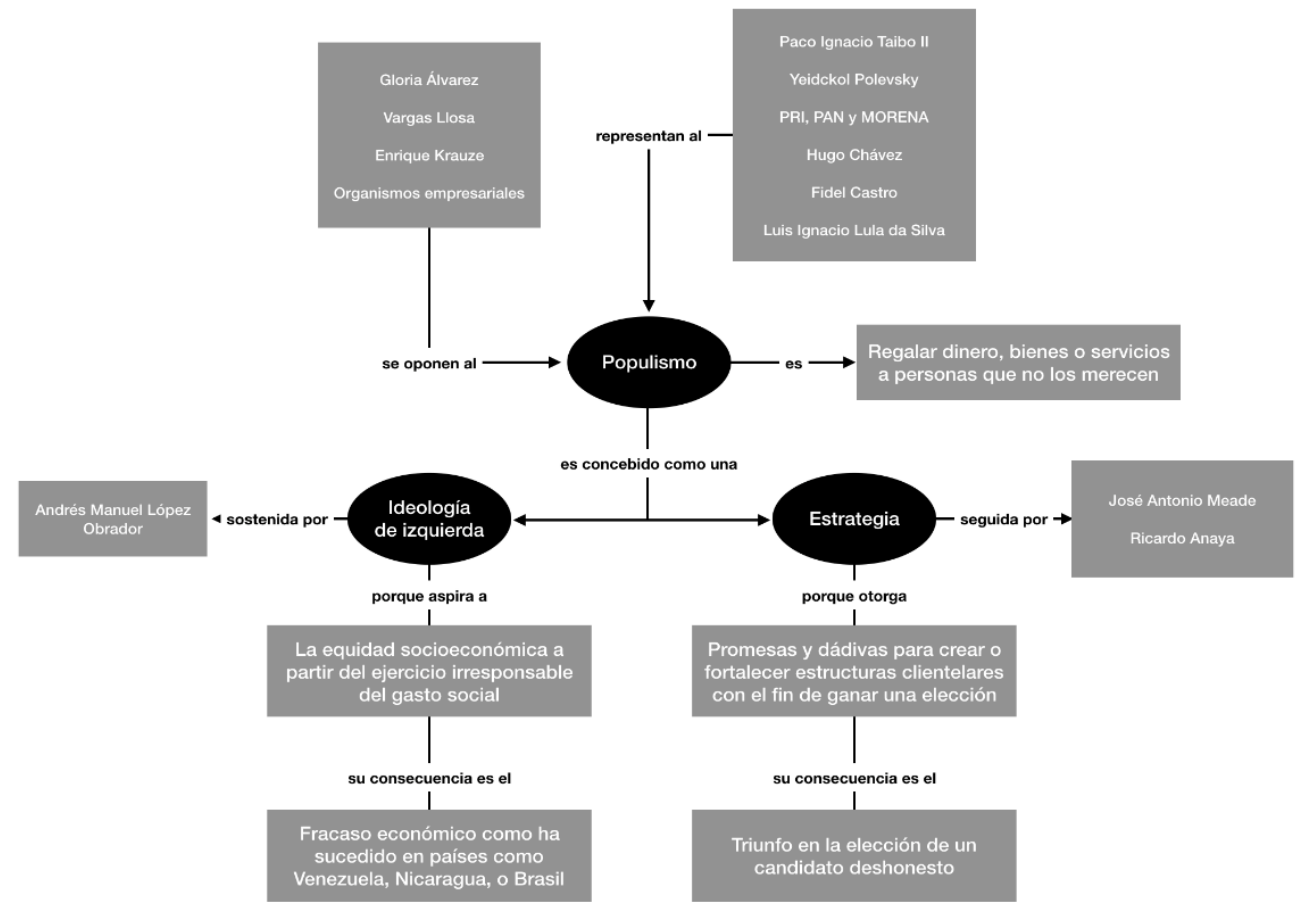

Se propone la ilustración de la Figura 2 a manera de modelo que describe la caracterización anterior. Por otra parte, la Figura 3 es una nube de palabras extraídas de los tuits que hicieron referencia al populismo, y que asimismo soportan el resumen que se propone.

Figura 3. Nube de palabras de tuits en que el populismo fue mencionado $(n=4,339)$

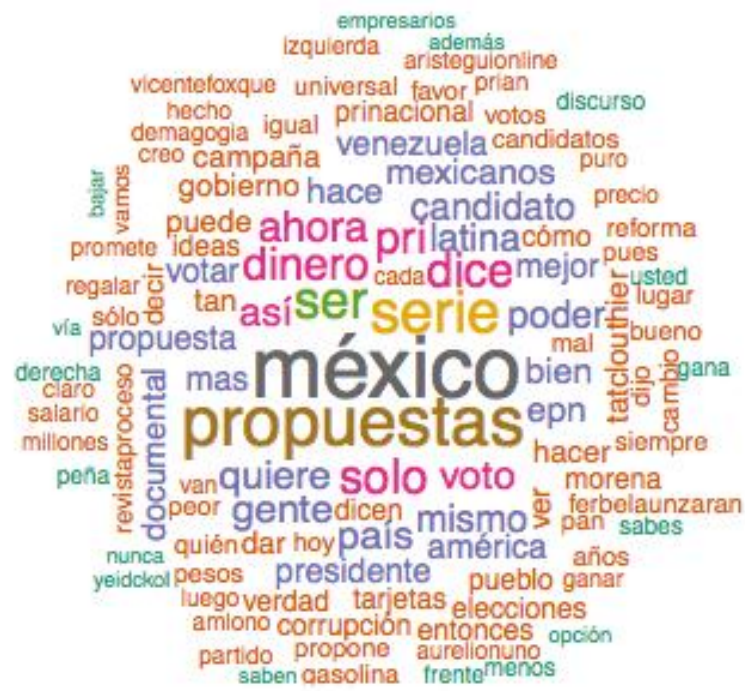

Nota: Se eliminaron de las posibles palabras los términos asociados a los nombres y apodos de los candidatos, así como aquellos relacionados con el populismo - populismo, populista, populistas-. Lo anterior con el fin de destacar en la nube las palabras definidoras del populismo. 


\section{Discusión}

El populismo en el contexto de la elección presidencial mexicana de 2018 fue definido por los usuarios de Twitter como una ideología y como una estrategia. Como comunicación, se identifican algunas características en la direccionalidad y contenido de los tuits que se agregan a la discusión teórica sobre cómo y desde dónde es definido este concepto.

Las aportaciones de los usuarios a la construcción de una definición concuerdan con la expectativa de que el populismo es una ideología raquítica (Mudde, 2004). Podemos observar en la conceptualización ideológica poca diversidad en la discusión temática; esto es, cuando se habla de populismo no se tocan posiciones concretas sobre género, medio ambiente, educación, o seguridad. El único tema relevante parece ser el económico, en el que se describe al populismo como proclive a realizar un gasto social irresponsable, con consecuencias catastróficas. Esta brevísima descripción de una política económica se relaciona con doctrinas no descritas (aunque sí mencionadas) por los usuarios que están ubicadas a la izquierda del continuo izquierda-derecha, tales como el comunismo o el socialismo. Lo anterior resultó congruente con la noción de que el populismo de izquierda prioriza en su discusión los temas económicos y la reivindicación del pueblo ante una crisis de inequidad (Lacatus, 2019). No obstante, esta asociación del populismo con la izquierda no tenía como fin el propuesto por Fredden (2017) de complementar los vacíos del populismo como propuesta, sino que los términos ideológicos fueron utilizados para descalificar las políticas económicas supuestamente sostenidas por uno de los candidatos. Para ilustrar esta descalificación, la crisis económica de algunos países que protagonizaron el giro a la izquierda en América Latina fue tomada como ejemplo de lo que podría pasar en México.

Como estrategia, el populismo es un término novedoso utilizado para designar a una serie de prácticas comunes en las elecciones mexicanas; concretamente la compra de votos y el clientelismo (e.g. Hilgers, 2008; Schedler, 2004), así como a la emisión de propuestas de baja calidad en tanto no permiten conocer las intenciones de largo plazo de los candidatos (Flores \& Flores, 2016). En este aspecto, hay coincidencia con la observación que hace Laclau (2004) respecto a que casi cualquier forma de política es populismo. Y también se respaldan las reflexiones de Weyland (2001) y Barr (2018) respecto a la apelación de las masas como fuente de poder, en tanto se percibe que, a través de dádivas y engaños, el objetivo político de los candidatos no es atraer el apoyo de grupos selectos, sino movilizar el apoyo de un gran número de personas vulnerables y poco organizadas.

Como comunicación, lo que mejor definió al populismo en las publicaciones de los usuarios fue la direccionalidad y el afecto negativo de su contenido. Las definiciones se hicieron desde la oposición al populismo y no desde su promoción. Siendo uno de los candidatos señalado como representante del populismo, los usuarios que simpatizaban con él respondían a esa designación con acusaciones de por qué los otros candidatos también eran populistas. Probablemente por esta razón, en contraposición con el concepto académico revisado, los usuarios no resaltaron la narrativa del pueblo 
agraviado por una élite. Antes bien, se habló sobre un agravio a las capacidades de los sectores productivos de la sociedad, clase media y empresarios, sobre quienes recaería el peso de pagar por los beneficios que recibe o recibiría un conjunto improductivo de personas.

Es importante reflexionar sobre lo anterior, debido a que el populismo como constructo académico parte a menudo de designaciones que se realizan en los medios y en el ámbito cotidiano. Algunos de los artículos en los que se discuten los componentes teóricos del populismo, han tomado en cuenta los señalamientos que hace la prensa de ciertos líderes como populistas, para justificar el análisis del comportamiento y discurso de estos líderes (e.g. Bolívar, 2017; Lacatus, 2019). A partir de los resultados que se presentan, es menester considerar que, en el ámbito de la comunicación, el populismo es un término que puede utilizarse estratégicamente para denostar algunas personalidades y plataformas políticas.

Es necesario aclarar que en este estudio usuarios de Twitter no quiere decir lo mismo que votantes, ciudadanía, o incluso -haciendo referencia a una categoría populista-, pueblo. En contradicción con su supuesta naturaleza horizontal, está ampliamente documentada la participación e influencia de las élites en las redes sociales en las discusiones electorales en México, no sólo por la alta participación de influencers y líderes de opinión, sino también a partir del uso de bots, trols y la divulgación de noticias falsas (e.g. Hernández, 2019; Magallón, 2019; Moo-Mena, Robles-Sandoval, González-Magaña, \& Rodríguez-Adame; 2019). La definición de populismo que se presenta en este trabajo no necesariamente es una construcción de los votantes.

Se concluye que a esta construcción subyace un concepto muy sencillo de enunciar, formado por una acción, de la que se espera una consecuencia. Populismo es la acción en la que una autoridad del estado da un beneficio gratuito a una persona o conjunto de personas que no merecen este beneficio, y se juzga que las consecuencias de otorgar este beneficio serán catastróficas para la economía del estado que lo otorga. Proponemos como temática de estudios futuros, indagar si este concepto y su uso como descalificativo se repite en otras discusiones electorales, más allá de las redes sociales, y más allá de Latinoamérica.

\section{Declaración de conflicto de intereses}

Los autores no informaron ningún posible conflicto de intereses.

\section{Referencias}

Aslanidis, P. (2016). Is populism an ideology? A refutation and a new perspective. Political Studies, 64(1S), 88-104. doi:10.1111/1467-9248.12224 
Balderas, R. G., \& Tapia, M. (2019). Algunos de los rasgos populistas de AMLO. El Cotidiano, 34(213), $28-36$.

Barr, R. (2018). Populism as a political strategy. En C. de La Torre (Ed.), Routledge International Handbook of Global Populism (pp. 44-56). Londres: Routledge.

Bedoya, J. T., \& Colín, A. (2016). El ciclo de vida del producto político: Estudio de caso Andrés Manuel López Obrador como candidato a la presidencia de México. Espacios Públicos, 19(45), 163181.

Bolívar, R. (2017). Liderazgo político: el caso de Andrés Manuel López Obrador en Movimiento de Regeneración Nacional (MORENA). Estudios Políticos, (42), 99-118.

Bulut, E., \& Yörük, E. (2017). Digital populism: Trolls and Political polarization of twitter in Turkey. International Journal of Communication, 11, 4093-4117.

Canovan, M. (2004). Populism for political theorists. Journal of Political Ideologies, 9(3), 241-252. doi:10.1080/1356931042000263500

Casero-Ripollés, A., Sintes-Olivella, M., \& Franch, P. (2017). The populist political communication style in action: Podemos's issues and functions on Twitter during the 2016 Spanish general election. American Behavioral Scientist, 61(9), 986-1001. doi:10.1177/0002764217707624.

Castañeda, J. (2006). Latin America's left turn. Foreign Affairs, 85(3), 28-43. doi:10.2307/20031965.

Engesser, S., Ernst, N., Esser, F., \& Büchel, F. (2017). Populism and social media: How politicians spread a fragmented ideology. Information, Communication \& Society, 2o(8), 1109-1126. doi:10.1080/1369118X.2016.1207697

Ernst, N., Engesser, S., Büchel, F., Blassnig, S., \& Esser, F. (2017). Extreme parties and populism: an analysis of Facebook and Twitter across six countries. Information, Communication \& Society, 2O(9), 1347-1364. doi:10.1080/1369118X.2017.1329333

Flores R. y Flores, J. C. (2016). En busca de la propuesta perdida. En R. A. Santana \& A. Aguayo (Eds.), Retratos Psicosociales en el México Contemporáneo (pp. 310-339). Guadalajara, MX: UVEDL.

Freeden, M. (2017). After the Brexit referendum: revisiting populism as an ideology. Journal of Political Ideologies, 22(1), 1-11. doi:10.1080/13569317.2016.1260813

Freidenberg, F. (2007). La tentación populista. Madrid, España: Síntesis.

Gerbaudo, P. (2018). Social media and populism: an elective affinity? Media, Culture \& Society, 4O(5), 745-753. doi:10.1177/1940161218790035

Groshek, J., \& Koc-Michalska, K. (2017). Helping populism win? Social media use, filter bubbles, and support for populist presidential candidates in the 2016 US election campaign. Information, Communication \& Society, 20(9), 1389-1407. doi:10.1080/1369118X.2017.1329334

Gutiérrez, S. (2007). La construcción de la imagen de López Obrador en los spots de sus adversarios. Cultura y Representaciones Sociales, 1(2), 31-54.

Hernández, M. (2019). La presencia de AMLO en Twitter en la precampaña de las elecciones federales de 2018 en México. Tesis de especialización, Universidad Veracruzana, México. 
Hilgers, T. (2008). Causes and consequences of political clientelism: Mexico's PRD in comparative perspective. Latin American Politics and Society, 5O(4), 123-153. doi:10.1111/j.15482456.2008.00032.x

Jacobs, K., \& Spierings, N. (2018). A populist paradise? Examining populists' Twitter adoption and use. Information, Communication \& Society, 1-16. doi:10.1080/1369118X.2018.1449883

Katsambekis, G., \& Stavrakakis, Y. (2017). Revisiting the nationalism/populism nexus: Lessons from the Greek case. Javnost - The Public, 24(4), 391-408. doi:10.1080/13183222.2017.1330087

Kearney, M. W. (2016). Rtweet : Collecting Twitter Data (Versión 0.1.7) [Software]. Recuperado de https://cran.r-project.org/package=rtweet

Lacatus, C. (2019). Populism and the 2016 American election: evidence from official press releases and twitter. Political Science \& Politics, 52(2), 223-228. doi:10.1017/S104909651800183X

Laclau, E. (2004). Populism, what's in a name? En B. Aretxaga, D. Dworkin, J. Gabilondo, \& J. Zulaika (Eds.), Empire and terror. Nationalism/postnationalism in the new millennium (pp. 103114). Nevada, US: Center for Basque Studies.

Loaeza, S. (2007). La desilusión mexicana. populismo y democracia en México en el 2006. Foro Internacional, 47(4), 817-838.

Magallón R. (2019). Verificado México 2018. Desinformación y fact-checking en campaña electoral. Revista de Comunicación, 18(1), 234-258. doi:10.26441/RC18.1-2019-A12

March, L., \& Mudde, C. (2005). What's left of the radical left? The European radical left after 1989: Decline and mutation. Comparative European Politics, 3(1), 23-49. doi:10.1057/palgrave.cep.6110052

Marchlewska, M., Cichocka, A., Panayiotou, O., Castellanos, K., \& Batayneh, J. (2018). Populism as identity politics: perceived in-group disadvantage, collective narcissism, and support for populism. Social Psychological and Personality Science, 9(2), 151-162. doi:10.1177/1948550617732393

Marini, A. M. (2018). El mesías tropical: aproximación a fenómenos populistas actuales a través del discurso de López Obrador. Chasqui. Revista Latinoamericana de Comunicación, 139, 153170. doi:10.16921/chasqui.voi139.3665

Mazzoleni, G. (2008). Populism and the media. En D. Albertazzi \& D. McDonell (Eds.), Twenty first century populism. The spectre of Western European democracy (pp. 49-64). New York, US: Palgrave Macmillan.

Moffitt, B., \& Tormey, S. (2014). Rethinking populism: Politics, mediatization and political style. Political Studies, 62(2), 381-397.doi:10.1111/1467-9248.12032

Moo-Mena, F., Robles-Sandoval, S., González-Magaña, K., \& Rodríguez-Adame, O. (2019). Towards bots detection by analyzing the behavior of user data on twitter. International Journal of Computer Science Issues (IJCSI), 16(1), 21-29. doi:10.5281/zenodo.2588241

Mudde, C. (2004). The populist zeitgeist. Government and Opposition, 39(4), 541-563. doi:10.1111/j.1477-7053.2004.00135.x

Mudde, C. (2010). The populist radical right: A pathological normalcy. West European Politics, 33(6), 1167-1186. doi:10.1080/01402382.2010.508901 
Ramiro, L., \& Gomez, R. (2017). Radical-left populism during the great recession: Podemos and its competition with the established radical left. Political Studies, 65(1S), 108-126. doi:10.1177/0032321716647400

Reinemann, C., Aalberg, T., Esser, F., Strömbäck, J., \& deVreese, C. H. (2016). Populist political communication: toward a model of its causes, forms, and effects. En T. Aalberg, F. Esser, C. Reinemann, J. Stromback, \& C. deVreese (Eds.), Populist Political Communication in Europe (pp. 22-36). New York, US: Routledge.

Rodrik, D. (2018). Is Populism necessarily bad economics? AEA Papers and Proceedings, 108, 196199. doi:10.1257/pandp.20181122.

Schedler, A. (2004). "El voto es nuestro": Cómo los ciudadanos mexicanos perciben el clientelismo electoral. Revista Mexicana de Sociología, 66(1), 57-97. doi: 10.22201/iis.01882503p.2004.001.5983

Stewart, J., Mazzoleni, G., \& Horsfield, B. (2003). Conclusion: Power to the media managers. En G. Mazzoleni, J. Stewart, \& B. Horsfield (Eds), The media and neopopulism: A contemporary comparative analysis (pp. 111-127). Westport, US: Praeger.

Urbinati, N. (2019). Political theory of populism. Annual Review of Political Science, 22(1), 111-127. doi:10.1146/annurev-polisci-050317-070753

Van Kessel, S., \& Castelein, R. (2016). Shifting the blame. Populist politicians' use of Twitter as a tool of opposition. Journal of Contemporary European Research, 12(2), 594-614.

Weyland, K. (2001). Clarifying a contested concept: Populism in the study of Latin American politics. Comparative Politics, 34(1), 1-22. doi:10.2307/422412 